\title{
Impacto do Fogo em Campo Sujo no Parque Estadual do Biribiri, Minas Gerais, Brasil
}

\author{
Milton Serpa de Meira Junior ${ }^{1}$, Israel Marinho Pereira ${ }^{2}$, \\ Evandro Luiz Mendonça Machado², Sílvia da Luz Lima Mota³ \\ Pedro Sousa Silva de Paula Ribeiro ${ }^{4}$, Thiago José Ornelas Otoni ${ }^{2}$
}

\author{
${ }^{1}$ Departamento de Engenharia Florestal, Universidade de Brasília - UnB, Brasília/DF, Brasil \\ ${ }^{2}$ Departamento de Engenharia Florestal, Universidade Federal dos Vales do Jequitinhonha e Mucuri - UFVJM, \\ Diamantina/MG, Brasil \\ ${ }^{3}$ Faculdade de Engenharia Florestal, Universidade Federal do Mato Grosso - UFMT, Cuiabá/MT, Brasil \\ ${ }^{4}$ Prefeitura Municipal de Paraisópolis, Paraisópolis/MG, Brasil
}

\section{RESUMO}

Objetivou-se avaliar a regeneração natural pós-fogo em uma área de campo sujo. Foram selecionados três ambientes; I e II, com ocorrência de fogo, e III, sem ocorrência. Em cada local foi instalado um transecto com 20 parcelas de $5 \times 5 \mathrm{~m}\left(25 \mathrm{~m}^{2}\right)$. Foram mensurados o diâmetro ao nível do solo (DNS), altura total e rebrota de todos os indivíduos com altura $\geq 0,10 \mathrm{~m}$ e DNS $\leq 5,0 \mathrm{~cm}$. Nos ambientes I e II foram amostrados 436 e 387 indivíduos e 29 e 24 espécies, respectivamente. Já no ambiente III foram amostrados 741 indivíduos e 29 espécies. Houve diferença significativa no número de perfilhos, número de indivíduos e na área basal. Nos ambientes I e II, 72\% e 88\%, respectivamente, apresentaram indícios de rebrotamento. O incêndio modificou a estrutura da vegetação e a rebrota foi a principal estratégia utilizada pelas espécies nos locais com ocorrência de incêndio.

Palavras-chave: cerrado, incêndio florestal, regeneração natural, ecologia do fogo.

\section{Dry Grassland Post-fire Impacts in Biribiri State Park, Minas Gerais, Brazil}

\begin{abstract}
This study aimed to evaluate the natural post-fire regeneration in dry grassland. We selected three sites: I and II, with occurrence of fire and III with no occurrence. Each site received one transect with 20 plots of $5 \times 5 \mathrm{~m}\left(25 \mathrm{~m}^{2}\right)$. Diameter at ground level (DNS), total height and regrowth of all individuals with height $\geq 0.10 \mathrm{~m}$ and DNS $\leq 5.0 \mathrm{~cm}$ were measured. Sites I and II, respectively, had 436 and 387 sampled individuals, and 29 and 24 species. Site III had 741 individuals and 29 species sampled. There was a significant difference in the number of tiller, individuals and basal area. In sites I and II, 72\% and $88 \%$, respectively, showed signs of regrowth. The fire changed the structure of vegetation, and regrowth was the main strategy used by species in sites with fire occurrence.
\end{abstract}

Keywords: Brazilian savanna, wild fire, natural regeneration, fire ecology. 


\section{INTRODUÇÃO}

O bioma Cerrado apresenta fisionomias que englobam formações florestais, savânicas e campestres. O termo campo designa áreas com predomínio de espécies herbáceas e algumas arbustivas, com poucas árvores formando paisagens abertas. Além do clima, da química e da física do solo, da disponibilidade de água e de nutrientes, da geomorfologia e da topografia, a distribuição da flora do Cerrado é condicionada pela latitude, pela frequência de queimadas, pela profundidade do lençol freático, pelo pastejo e por inúmeros fatores antrópicos (Ribeiro \& Walter, 2008).

Durante o período sazonal de secas típico das regiões de cerrado, o estrato herbáceo e as gramíneas estão, de forma generalizada, latentes, apresentando grande percentagem de sua biomassa seca, favorecendo a ocorrência de incêndios (Klink \& Solbrig, 1996). Dentre os vários distúrbios naturais e antrópicos que desencadeiam o processo de alteração, os incêndios podem influenciar a sucessão vegetal, a dinâmica da floresta, o acúmulo de serrapilheira, a ciclagem de nutrientes, a população de insetos, o banco de sementes no solo e a taxa de rebrota de espécies arbustivo-arbóreas (Kauffman, 1991; Cochrane \& Schulze, 1999). Posteriormente, a perpetuação dos indivíduos da regeneração depende de fatores fenológicos, genéticos e ambientais (Felfili \& Rezende, 2003) e de distúrbios ocasionais, como fogo (Hoffmann, 1998).

Os efeitos do fogo na vegetação e suas consequências na riqueza das espécies e no estabelecimento das plântulas, bem como sua resiliência, tolerância, resistência, dependência ou sensibilidade em relação às suas variações de sítios e intensidade ainda são muito questionados, dividindo a comunidade científica em diferentes linhas de pensamento. Nesse sentido, o presente trabalho teve como objetivo avaliar a capacidade de rebrota e o potencial da regeneração natural pós-fogo em uma área de campo sujo.

\section{MATERIAL E MÉTODOS}

\subsection{Localização e caracterização da área de estudo}

$O$ estudo foi realizado em um trecho do Parque Estadual do Biribiri (PEB) inserido no complexo da Serra do Espinhaço, no município de abrangência de
Diamantina, Estado de Minas Gerais. O parque encontra-se localizado nas coordenadas $18^{\circ} 12^{\prime} 18^{\prime \prime}-18^{\circ} 12^{\prime} 17^{\prime \prime} \mathrm{S}$ e $43^{\circ} 34^{\prime} 55^{\prime \prime}-43^{\circ} 35^{\prime} 05^{\prime \prime} \mathrm{W}$, com altitude de $1.395 \mathrm{~m}$. O clima é classificado como Cwb, segundo a classificação de Köppen, temperado úmido, com inverno seco e chuvas no verão, com precipitação média anual de $1.400 \mathrm{~mm}$, temperaturas amenas durante todo ano (média anual em torno de $19^{\circ} \mathrm{C}$ a $18^{\circ} \mathrm{C}$ ) (Neves et al., 2005).

Fisionomicamente, o PEB caracteriza-se pelo predomínio de formações savânicas (cerrados sentido restrito e rupestre), campestres (campos limpos, úmidos e rupestres) e florestais (florestas estacionais semideciduais), constituindo um ecótone entre o Cerrado e a Mata Atlântica (Pereira et al., 2015). As áreas de vegetação campestre são constantemente afetadas pelos incêndios florestais no cerrado (Miranda et al., 2002). O PEB, entre os anos de 2007 e 2011, teve uma área de 1.958,21 hectares atingida por incêndios florestais (Ávila \& Souza, 2012).

\subsection{Amostragem da vegetação regenerante}

O trabalho foi realizado em uma área com ocorrência de incêndio, sendo o inventário realizado cinco meses após a ocorrência, logo após o período chuvoso, quando a regeneração natural começa a colonizar novamente a área. Para amostragem da vegetação regenerante foram selecionados, na área de interesse, três ambientes; sendo dois com influência do distúrbio (I e II) e um sem esse fator (III). Em cada um dos ambientes foi instalada uma parcela com dimensões de $10 \times 50 \mathrm{~m}$ $\left(500 \mathrm{~m}^{2}\right)$, subdividida em 20 subparcelas de $5 \times 5 \mathrm{~m}$ $\left(25 \mathrm{~m}^{2}\right)$ (Figura 1). No interior de cada subparcela

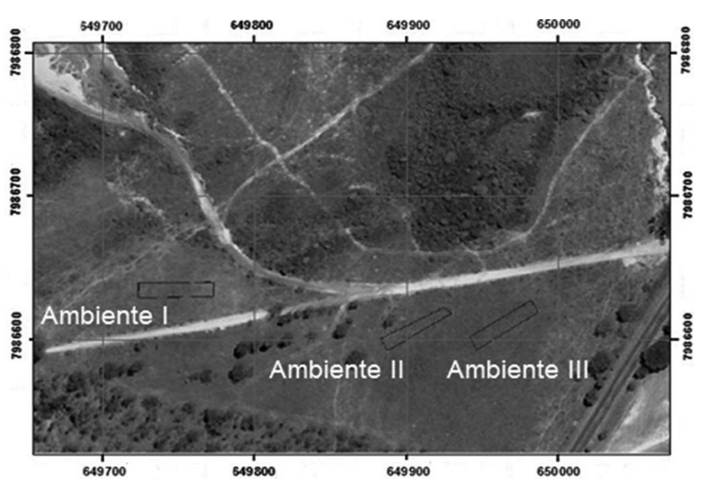

Figura 1. Localização das unidades amostrais no Parque Estadual do Biribiri, Minas Gerais, Brasil.

Figure 1. Location of the sampling units in the Biribiri State Park, Minas Gerais Brazil. 
foram amostrados todos os indivíduos vivos da flora arbustivo-arbórea com altura $>0,10 \mathrm{~m}$ e diâmetro ao nível do solo (DNS) $<5,0 \mathrm{~cm}$. Foram avaliados para cada indivíduo o DNS, altura total e ocorrência de rebrotas, consideradas quando ocorreram na base do caule ou cepas.

Os indivíduos foram distribuídos em três classes de tamanho de regeneração natural, segundo metodologia recomendada por Finol (1971), adaptada para este trabalho: Classe 1: indivíduos com altura entre 0,10 e 0,30 m; Classe 2: indivíduos com altura entre 0,31 e 1,50 m; e Classe 3: indivíduos com altura superior a $1,50 \mathrm{~m}$.

O material botânico testemunho foi herborizado e depositado no Herbário Dendrológico Jeanine Felfili (HDJF), pertencente à Universidade Federal dos Vales do Jequitinhonha e Mucuri, no Departamento de Engenharia Florestal. As identificações foram feitas com base na literatura especializada (Lorenzi, 1992; Silva-Júnior, 2005; Souza \& Lorenzi, 2005), consultas a especialistas e coleções do herbário. As espécies foram classificadas nas famílias reconhecidas pelo sistema do Angiosperm Phylogeny Group III (APG III, 2009).

Para análise da estrutura da regeneração natural foram utilizados os parâmetros clássicos descritos por Mueller-Dombois \& Ellenberg (1974): Densidade, Dominância e Frequência em valores absolutos e relativos e o Índice de Valor de Importância (IVI). Também foram calculadas as estimativas da Regeneração Natural (RN), conforme proposta por Finol (1971). As classes de tamanho adotadas foram: Classe I: indivíduos com altura entre $0,1 \mathrm{~m}$ e $0,3 \mathrm{~m}$; Classe II: indivíduos com altura entre 0,31 m e 1,51 m e; Classe III: indivíduos com altura superior a $1,51 \mathrm{~m}$.

A diversidade foi mensurada dentro (alfa) e entre os ambientes (beta). $\mathrm{O}$ índice de diversidade de Shannon (H' - Equação 1) e a Equabilidade de Pielou (J' - Equação 2) foram utilizados para mensurar a diversidade alfa (Brower \& Zar, 1984). Já o índice de similaridade de Jaccard (SJ - Equação 3) quantitativo e o diagrama de Venn, para mensurar beta.

$$
\begin{aligned}
H^{\prime} & =-\sum_{i=1}^{n} p i \times \ln p i \quad p i=N i / N T \\
J^{\prime} & =\frac{H^{\prime}}{\ln S} \\
S J & =\frac{A+B+C}{C}
\end{aligned}
$$

em que: $\mathrm{Ni}$ = número de indivíduos da i-ésima espécie; $\mathrm{NT}=$ número total de indivíduos amostrados; $\mathrm{S}=$ número de espécies; $\mathrm{A}=$ riqueza de espécie da comunidade $\mathrm{A}$; $\mathrm{B}=$ riqueza de espécie da comunidade $\mathrm{B} ; \mathrm{C}=$ número de espécies em comum das comunidades A e B.

\subsection{Análise estatística}

Utilizou-se análise de variância para comparar as estruturas: número de indivíduos total, número médio de perfilhos e área basal entre os ambientes. Para comparar o número de indivíduos entre as classes de tamanho foi utilizada a análise de variância com dois fatores. Todas as análises foram efetuadas utilizando-se o pacote ExpDes, desenvolvido por Ferreira et al. (2013) no software R 3.2.1 (R Development Core Team, 2015).

\section{RESULTADOS E DISCUSSÕES}

Nos três ambientes estudados foram registrados 1.564 indivíduos arbustivo-arbóreos regenerantes, representados por 22 famílias, 33 gêneros e 53 espécies. Nos ambientes com ocorrência de fogo I e II foram amostrados 436 (27,87\%) e 387 (24,75\%) indivíduos e 29 e 24 espécies, respectivamente. Já no ambiente III, que não foi atingido pelo fogo, foram amostrados 741 (47,38\%) indivíduos e 29 espécies (Tabela 1).

De modo geral, as famílias que apresentaram maior número de espécies nos ambientes com ocorrência de fogo foram Myrtaceae (10), Asteraceae (6), Fabaceae (5), Malpighiaceae (5) e Bignoniaceae (4), contribuindo juntas com $54,71 \%$ do total de espécies amostradas. As famílias Myrtaceae, Asteraceae, Fabaceae, Bignoniaceae que se destacaram em riqueza nos ambientes com ocorrência de fogo têm sido comumente apontadas entre as mais ricas em diversos estudos da flora do Cerrado (sensu lato) (Leitão-Filho, 1992; Ratter et al., 1996; Felfili et al., 1998; Mendonça et al., 1998; Tannus \& Assis, 2004), indicando que a ocorrência de fogo não modificou a florística das famílias nesse ambiente.

O índice de semelhança de Jaccard e o diagrama de Venm (Figura 2) mostram que os ambientes I e III são os mais similares quantitativamente, mas em termos qualitativos os ambientes mais semelhantes foram I e II. Não há parâmetro suficiente para afirmar que o fogo modificou qualitativamente e quantitativamente a composição florística no ambiente III. 
Tabela 1. Relação de espécies amostradas no campo sujo em Diamantina, MG, com respectivos parâmetros fitossociológicos para os ambientes I, II e III, onde: $\mathrm{Ni}$ = número de indivíduos da i-ésima; IVI = índice de valor de importância (\%); $\mathrm{RN}$ = estimativa da regeneração natural relativa.

Table 1. List of species sampled in the shrub in Diamantina-MG, with their respective environments phytosociological parameters for site I, II and III, where: $\mathrm{Ni}=$ number of individuals of the i th; IVI = importance value index (\%); $\mathrm{RN}=$ estimate of natural regeneration.

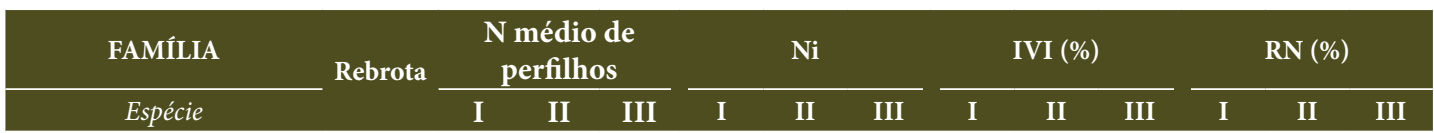

\section{ANNONACEAE}

\begin{tabular}{|c|c|c|c|c|c|c|c|c|c|c|c|c|c|}
\hline Annona sp. & $\mathrm{X}$ & - & 2 & 1 & - & 2 & 1 & - & 0,71 & 0,28 & - & 1,84 & 1,6 \\
\hline \multicolumn{14}{|l|}{ AQUIFOLIACEAE } \\
\hline Ilex cerasifolia Reissek & $\mathrm{X}$ & - & 4,5 & - & - & 2 & - & - & 0,95 & - & - & 2,16 & - \\
\hline Ilex sp. & $\mathrm{X}$ & 1 & 1 & - & 1 & 1 & - & 0,16 & 0,54 & - & 0,38 & 1,96 & - \\
\hline \multicolumn{14}{|l|}{ ASTERACEAE } \\
\hline Asteraceae 1 & $\mathrm{X}$ & - & 9,71 & 1,75 & - & 14 & 16 & - & 5,26 & 2,63 & - & 5,23 & 3,57 \\
\hline $\begin{array}{l}\text { Baccharis brachylaenoides } \\
\text { DC. }\end{array}$ & & - & - & 1 & - & - & 1 & - & - & 0,31 & - & - & 0,29 \\
\hline Bacharis sp. & & 4,5 & 2 & - & 4 & 2 & - & 0,91 & 0,96 & - & 1,7 & 2,16 & - \\
\hline $\begin{array}{l}\text { Eremanthus glomerulatus } \\
\text { Less. }\end{array}$ & $\mathrm{X}$ & 3 & - & - & 1 & - & - & 0,2 & - & - & 0,41 & - & - \\
\hline $\begin{array}{l}\text { Eremanthus incanus } \\
\text { (Less.) Less. }\end{array}$ & & 2 & - & - & 1 & - & - & 0,2 & - & - & 0,41 & - & - \\
\hline Gochnatia pulchra Cabrera & & - & 1 & - & - & 1 & - & - & 0,45 & - & - & 1,96 & - \\
\hline \multicolumn{14}{|l|}{ BIGNONIACEAE } \\
\hline $\begin{array}{l}\text { Jacaranda caroba (Vell.) } \\
\text { A. DC. Cham. }\end{array}$ & $\mathrm{X}$ & 1 & 1,46 & 1 & 4 & 28 & 13 & 0,81 & 7,03 & 2,85 & 1,09 & 6,37 & 3,87 \\
\hline $\begin{array}{l}\text { Handroanthus ochraceos } \\
\text { (Cham.) Standl. }\end{array}$ & $\mathrm{X}$ & 1,06 & - & 1 & 79 & - & 1 & 16,4 & - & 0,33 & 14,76 & - & 1,6 \\
\hline Zeyheria montana Mart. & $\mathrm{X}$ & 1 & - & 1 & 1 & - & 9 & 0,26 & - & 1,62 & 0,45 & - & 2,06 \\
\hline \multicolumn{14}{|l|}{ CHRYSOBALANACEAE } \\
\hline $\begin{array}{l}\text { Couepia grandiflora (Mart. } \\
\text { e Zucc.) Benth. ex Hook. f. }\end{array}$ & & 2,2 & - & 1 & 5 & - & 7 & 1,01 & - & 1,24 & 1,22 & - & 2,17 \\
\hline \multicolumn{14}{|l|}{ CLUSIACEAE } \\
\hline $\begin{array}{l}\text { Kielmeyera coriacea Mart. } \\
\text { e Zucc. }\end{array}$ & & - & - & 1,21 & - & - & 149 & - & - & 20,17 & - & - & 10,71 \\
\hline $\begin{array}{l}\text { Kielmeyera lathrophyton } \\
\text { Saddi }\end{array}$ & $\mathrm{X}$ & - & 1 & - & - & 1 & - & - & 0,49 & - & - & 1,96 & - \\
\hline \multicolumn{14}{|l|}{ ERYTHROXYLACEAE } \\
\hline $\begin{array}{l}\text { Erythroxylum campestre } \\
\text { St. Hil. }\end{array}$ & & - & - & 7 & - & - & 1 & - & - & 0,32 & - & - & 1,6 \\
\hline $\begin{array}{l}\text { Erythroxylum cuneifolium } \\
\text { (Mart.) O. E. Schulz }\end{array}$ & & - & - & 2 & - & - & 1 & - & - & 0,28 & - & - & 1,6 \\
\hline $\begin{array}{l}\text { Erythroxylum suberosum } \\
\text { A. St.-Hil. }\end{array}$ & $\mathrm{X}$ & - & 1,41 & 1,4 & - & 27 & 5 & - & 9,37 & 1,8 & - & 7,51 & 2,64 \\
\hline $\begin{array}{l}\text { Erythroxylum tortuosum } \\
\text { Mart. }\end{array}$ & $\mathrm{X}$ & - & 1,3 & 1 & - & 23 & 18 & - & 4,77 & 3,93 & - & 4,25 & 3,49 \\
\hline \multicolumn{14}{|l|}{ EUPHORBIACEAE } \\
\hline Croton sp. & & - & - & 1 & - & - & 1 & - & - & 0,27 & - & - & 1,6 \\
\hline $\begin{array}{l}\text { Sapium glandulosum (L.) } \\
\text { Morong }\end{array}$ & $\mathrm{X}$ & - & 7,25 & - & - & 4 & - & - & 1,97 & - & - & 3,15 & - \\
\hline \multicolumn{14}{|l|}{$\begin{array}{l}\text { FABACEAE CAESAL- } \\
\text { PINIOIDEAE }\end{array}$} \\
\hline $\begin{array}{l}\text { Senna multijuga } \\
\text { (L. C. Rich.) H. S. Irwin e } \\
\text { Barneby }\end{array}$ & & - & - & 1 & - & - & 1 & - & - & 0,28 & - & - & 1,6 \\
\hline
\end{tabular}


Tabela 1. Continuação...

Table 1. Continued...

\begin{tabular}{|c|c|c|c|c|c|c|c|c|c|c|c|c|c|}
\hline \multirow{2}{*}{$\begin{array}{c}\text { FAMÍLIA } \\
\text { Espécie }\end{array}$} & \multirow[t]{2}{*}{ Rebrota } & \multicolumn{3}{|c|}{$\begin{array}{c}\mathbf{N} \text { médio de } \\
\text { perfilhos }\end{array}$} & \multicolumn{3}{|c|}{$\mathbf{N i}$} & \multicolumn{3}{|c|}{ IVI (\%) } & \multicolumn{3}{|c|}{ RN (\%) } \\
\hline & & I & II & III & I & II & III & I & II & III & I & II & III \\
\hline $\begin{array}{l}\text { Senna silvestris (Vell.) } \\
\text { H. S. Irwin e Barneby }\end{array}$ & $\mathrm{X}$ & 3 & - & 0 & 7 & - & - & 1,86 & - & - & 2,34 & - & - \\
\hline \multicolumn{14}{|l|}{$\begin{array}{l}\text { FABACEAE } \\
\text { FABOIDEAE }\end{array}$} \\
\hline $\begin{array}{l}\text { Clitoria guianensis (Aubl.) } \\
\text { Benth. }\end{array}$ & $\mathrm{X}$ & 2 & - & - & 2 & - & - & 0,36 & - & - & 0,79 & - & - \\
\hline Crotalaria sp. & $\mathrm{X}$ & 1 & - & - & 2 & - & - & 0,36 & - & - & 0,79 & - & - \\
\hline $\begin{array}{l}\text { Dalbergia miscolobium } \\
\text { Benth. }\end{array}$ & $\mathrm{X}$ & 7,32 & 3,7 & 1,76 & 22 & 10 & 29 & 10,8 & 4,13 & 5,62 & 11,03 & 3,84 & 4,71 \\
\hline \multicolumn{14}{|l|}{ LAMIACEAE } \\
\hline Hyptis crinita Benth. & & - & - & 1,33 & - & - & 3 & - & - & 0,44 & - & - & 1,69 \\
\hline \multicolumn{14}{|l|}{ MALPIGHIACEAE } \\
\hline $\begin{array}{l}\text { Banisteriopsis campestris } \\
\text { A. Jun. }\end{array}$ & $\mathrm{X}$ & 2,15 & 2,18 & 1 & 92 & 11 & 11 & 17,7 & 3,67 & 2,39 & 16,99 & 4,38 & 3,56 \\
\hline $\begin{array}{l}\text { Byrsonima coccolobifolia } \\
\text { Kunth. }\end{array}$ & $\mathrm{X}$ & 4,47 & 1 & - & 30 & 2 & - & 6,93 & 0,93 & - & 6,53 & 1,96 & - \\
\hline $\begin{array}{l}\text { Byrsonima guilleminiana } \\
\text { A. Juss. }\end{array}$ & $\mathrm{X}$ & 8 & - & - & 1 & - & - & 0,27 & - & - & 0,46 & - & - \\
\hline $\begin{array}{l}\text { Byrsonima intermedia } \\
\text { A. Juss. }\end{array}$ & & - & - & 1,23 & - & - & 40 & - & - & 4,3 & - & - & 4,86 \\
\hline \multicolumn{14}{|l|}{ MELIACEAE } \\
\hline $\begin{array}{l}\text { Cabralea canjerana (Vell.) } \\
\text { Mart. }\end{array}$ & $\mathrm{X}$ & 2 & - & - & 1 & - & - & 0,31 & - & - & 0,48 & - & - \\
\hline \multicolumn{14}{|l|}{ MYRSINACEAE } \\
\hline $\begin{array}{l}\text { Myrsine guianensis (Aubl.) } \\
\text { Kuntze. }\end{array}$ & $\mathrm{X}$ & 2,01 & 2,22 & 1,5 & 78 & 112 & 304 & 17,03 & 22,41 & 31,83 & 13,54 & 13,55 & 19,25 \\
\hline \multicolumn{14}{|l|}{ MYRTACEAE } \\
\hline $\begin{array}{l}\text { Campomanesia } \\
\text { adamantium (Cambess.) } \\
\text { O. Berg }\end{array}$ & $\mathrm{X}$ & - & 2,8 & - & - & 5 & - & - & 1,27 & - & - & 2,29 & - \\
\hline Eugenia bimarginata D. C. & $\mathrm{X}$ & 3 & 5 & 1 & 1 & 1 & 3 & 0,17 & 0,47 & 0,6 & 0,38 & 1,96 & 1,91 \\
\hline Eugenia observa O. Berg & & 3 & - & - & 2 & - & - & 0,54 & - & - & 0,63 & - & - \\
\hline $\begin{array}{l}\text { Eugenia punicifolia } \\
\text { (Kunth) DC. }\end{array}$ & $\mathrm{X}$ & 4,85 & 4,49 & 1,89 & 20 & 108 & 53 & 4,98 & 23,57 & 6,32 & 4,68 & 16,82 & 6,51 \\
\hline $\begin{array}{l}\text { Eugenia sonderiana } \\
\text { O. Berg }\end{array}$ & & - & - & 1 & - & - & 3 & - & - & 0,63 & - & - & 1,91 \\
\hline Gomidesia sp. & & 5 & - & - & 3 & - & - & 1,01 & - & - & 1,22 & - & - \\
\hline Myrtaceae 1 & & - & - & 1,05 & - & - & 19 & - & - & 2,44 & - & - & 3,49 \\
\hline Myrtaceae 2 & & - & - & 1 & - & - & 1 & - & - & 0,27 & - & - & 0,38 \\
\hline Psidium guineense Sw. & & 4,7 & - & - & 10 & - & - & 2,08 & - & - & 2,48 & - & - \\
\hline $\begin{array}{l}\text { Psidium cinereum } \\
\text { Mart. ex DC. }\end{array}$ & $\mathrm{X}$ & 2,33 & 2 & - & 3 & 2 & - & 0,64 & 0,89 & - & 0,97 & 1,96 & - \\
\hline Psidium sp. & & - & - & 1 & - & - & 13 & - & - & 2,69 & - & - & 3,68 \\
\hline \multicolumn{14}{|l|}{ OCHNACEAE } \\
\hline Ouratea floribunda Engl. & $\mathrm{X}$ & - & 2 & - & - & 1 & - & - & 0,46 & - & - & 1,96 & - \\
\hline \multicolumn{14}{|l|}{ POLYGONACEAE } \\
\hline $\begin{array}{l}\text { Coccoloba brasiliensis Nees } \\
\text { e Mart. }\end{array}$ & $\mathrm{X}$ & 3 & 3 & - & 2 & 1 & - & 0,96 & 0,44 & - & 0,91 & 1,56 & - \\
\hline \multicolumn{14}{|l|}{ RUBIACEAE } \\
\hline Palicourea rigida Kunth. & $\mathrm{X}$ & 1 & 1,67 & 1 & 1 & 3 & 1 & 0,23 & 1,07 & 0,33 & 0,43 & 2,18 & 1,6 \\
\hline
\end{tabular}


Tabela 1. Continuação...

Table 1. Continued...

\begin{tabular}{|c|c|c|c|c|c|c|c|c|c|c|c|c|c|}
\hline \multirow{2}{*}{$\begin{array}{c}\text { FAMÍLIA } \\
\text { Espécie }\end{array}$} & \multirow{2}{*}{ Rebrota } & \multicolumn{3}{|c|}{$\begin{array}{c}\mathbf{N} \text { médio de } \\
\text { perfillos }\end{array}$} & \multicolumn{3}{|c|}{$\mathbf{N i}$} & \multicolumn{3}{|c|}{ IVI (\%) } & \multicolumn{3}{|c|}{ RN (\%) } \\
\hline & & $\mathbf{I}$ & II & III & $\mathbf{I}$ & II & III & I & II & III & $\bar{I}$ & II & III \\
\hline \multicolumn{14}{|l|}{ SALICACEAE } \\
\hline Casearia rupestris Eichler. & & 1,45 & - & - & 1 & - & - & 0,23 & - & - & 0,43 & - & - \\
\hline Casearia sylvestris Sw. & $\mathrm{X}$ & 1,45 & 1,95 & 1,25 & 29 & 21 & 16 & 5,81 & 5,83 & 2,66 & 6,06 & 5,56 & 3,09 \\
\hline
\end{tabular}

\section{SAPINDACEAE}

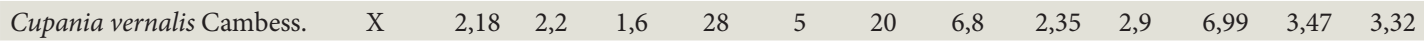

\section{SOLANACEAE}

\begin{tabular}{lcccccccccccc}
$\begin{array}{l}\text { Solanum grandiflorum } \\
\text { Desf. }\end{array}$ & 1 & - & - & 5 & - & - & 0,97 & - & - & 1,47 & - & - \\
VERBENACEAE & & & & & & & & & & & \\
Lipia sp. & - & - & 3 & - & - & 1 & - & - & 0,28 & - & - & 1,6 \\
Soma/média & $\mathbf{2 , 7 8}$ & $\mathbf{2 , 7 2}$ & $\mathbf{1 , 4 8}$ & $\mathbf{4 3 6}$ & $\mathbf{3 8 7}$ & $\mathbf{7 4 1}$ & $\mathbf{1 0 0}$ & $\mathbf{1 0 0}$ & $\mathbf{1 0 0}$ & $\mathbf{1 0 0}$ & $\mathbf{1 0 0}$ & $\mathbf{1 0 0}$ \\
\hline
\end{tabular}

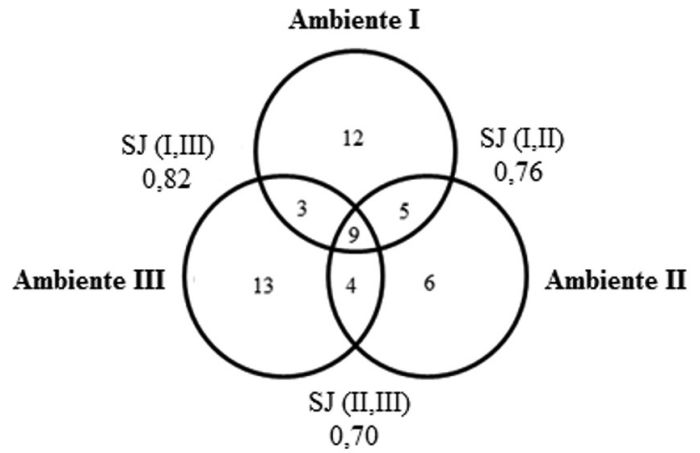

Figura 2. Valores do índice de semelhança de Jaccard (J) e distribuição do número de espécies em três ambientes de Cerrado com (I e II) e sem ocorrência de fogo (III), em Diamantina, MG.

Figure 2. Values of Jaccard similarity index $(J)$ and the distribution of the number of species in three savannah ambient with (I and II) and without (III) the occurrence of fire in Diamantina, MG.

Dentre as 39 espécies registradas para os três ambientes estudados, M. guianensis (Myrsinaceae) foi a que apresentou maior número de indivíduos, representando $23,08 \%$ do total amostrado, seguida de E. punicifolia (15,55\%), B. campestris (12,52\%) e H. ochraceos $(9,60 \%)$, totalizando $60,75 \%$ dos indivíduos amostrados.

As espécies de maior RN no ambiente I foram B. campestris $(16,99 \%), H$. ochraceus $(14,76 \%)$, M. guianensis $(13,54 \%)$, D. miscolobium $(11,03 \%)$ e B. coccolobifolia (6,53\%), as quais juntas somam $69,54 \%$ da RN e 75,65\% do IVI. No ambiente II, as espécies de maior RN foram E. punicifolia (16,82\%), M. guianensis (13,55\%), E. suberosum (7,51\%), J. caroba $(6,37 \%)$ e
C. sylvestris (5,56\%), que juntas somam $49,80 \%$ da RN e 68,2\% do IVI. Já no ambiente III, as espécies de maior RN foram M. guianensis (19,25\%), K. coriaceae (10,71\%), E. punicifolia (6,51\%), B. intermedia (4,86\%) e D. miscolobium (4,71\%), que somadas representaram pouco mais de $46 \%$ da $\mathrm{RN}$ e $68 \%$ do IVI.

Não houve diferença na estrutura horizontal da regeneração natural entre os ambientes, rejeitando-se a hipótese de que o efeito do fogo alteraria a estrutura da comunidade. Fato semelhante ocorreu nos trabalhos sobre matas de galeria (Nascimento, 1996; Martins et al., 2002; Santiago et al., 2005). Entretanto, Xavier et al. (2009) identificaram diferença na estrutura da regeneração natural devida a efeito do fogo.

O índice de diversidade de Shannon ( $\left.H^{\prime}\right)$ encontrado nos ambientes I; II; e III foi de 2,88; 2,55; e 3,13 nats. ind $^{-1}$, respectivamente. $\mathrm{O}$ índice de equabilidade de Pielou (J') encontrado nos ambientes I; II; e III foi de 0,$86 ; 0,80$; e 0,93 , respectivamente. Na ocorrência de fogo prevalece o desenvolvimento de espécies que são resilientes ao incêndio, e como esse número de espécies é pequeno, a diversidade é consequentemente reduzida e a homogeneidade local, maior.

Nos ambientes I e II, 72\% e 88\%dos indivíduos, respectivamente, apresentaram indícios de rebrotamento, ou seja, a rebrota é a principal estratégia das espécies na colonização de ambientes após a ocorrência de fogo (Nascimento, 1996; Martins et al., 2002; Xavier et al., 2011). Segundo Santos et al. (2003), as espécies dominantes em locais com ocorrência de fogo apresentam a estratégia de rebrota. 
Tabela 2. Valores médios de riqueza e abundância para três ambientes de Cerrado com e sem ocorrência de fogo em Diamantina, MG. NIT = número de indivíduos total; NIC1 = número de indivíduos na classe 1; NIC2 = número de indivíduos na classe 2; NIC3 = número de indivíduos na classe 3; NP = número de perfilhos.

Table 2. Mean values of wealth and abundance for three savannah ambient with and without the occurrence of fire in Diamatina, MG. Which: NIT = total number of individuals; NICI = number of individuals in class I; $\mathrm{NICII}=$ number of individuals in class II; NICIII = number of individuals in class III; NP = number of tiller.

\begin{tabular}{|c|c|c|c|c|c|}
\hline FV & GL & SQ & QM & $\mathbf{F}$ & P-valor \\
\hline Ambiente & 2 & 1230,8 & 615,4 & 13,066 & $2.1 \mathrm{e}-05$ \\
\hline Erro a & 57 & 2684,8 & 47,1 & & \\
\hline Classe de altura & 2 & 10492,4 & 5246,2 & 147,229 & $2 \mathrm{e}-16$ \\
\hline Ambiente $^{\star}$ Classe de Altura & 4 & 5490,7 & 1372,7 & 38,523 & $2 \mathrm{e}-16$ \\
\hline Erro b & 114 & 4062,2 & 35,6 & & \\
\hline Total & 179 & 23961,0 & & & \\
\hline FV & GL & SQ & QM & $\mathbf{F}$ & P-valor \\
\hline Ambiente: Classe de Altura 1 & 2 & 445,3 & 222,65 & 5,643 & 0,004248 \\
\hline Ambiente: Classe de Altura 2 & 2 & 6272,13 & 3136,07 & 79,48 & $2 e-16$ \\
\hline Ambiente: Classe de Altura 3 & 2 & 4,13 & 2,067 & 0,052 & 0,949 \\
\hline Pooled Error & 167 & 6622,61 & 39,46 & 38,523 & \\
\hline Ambiente & \multicolumn{2}{|c|}{ I } & II & \multicolumn{2}{|r|}{ III } \\
\hline NIT & \multicolumn{2}{|c|}{$436^{\mathrm{b}}$} & $387^{\mathrm{b}}$ & \multicolumn{2}{|r|}{$741^{\mathrm{a}}$} \\
\hline NP & \multicolumn{2}{|c|}{$2,513^{b}$} & $3,325^{\mathrm{a}}$ & \multicolumn{2}{|r|}{$1,427^{c}$} \\
\hline Área Basal & \multicolumn{2}{|c|}{$0,0267^{b}$} & $0,0286^{\mathrm{b}}$ & \multicolumn{2}{|r|}{$0,0977^{a}$} \\
\hline NIC1 & \multicolumn{2}{|c|}{$183^{\mathrm{a}}$} & $162^{a}$ & \multicolumn{2}{|r|}{$58^{\mathrm{b}}$} \\
\hline NIC2 & \multicolumn{2}{|c|}{$248^{\mathrm{b}}$} & $218^{\mathrm{b}}$ & \multicolumn{2}{|r|}{$666^{a}$} \\
\hline NIC3 & \multicolumn{2}{|c|}{$5^{\mathrm{a}}$} & $7^{a}$ & \multicolumn{2}{|r|}{$17^{\mathrm{a}}$} \\
\hline
\end{tabular}

Médias seguidas por letras diferentes indicam diferença significativa entre os ambientes a $5 \%$ de probabilidade pelo teste de Tukey.

Houve diferença significativa, a 5\% de significância, entre os parâmetros número de indivíduos total, indivíduos na classe 1 , indivíduos na classe 2 , indivíduos na classe 3, número de perfilho e área basal (Tabela 2).

Os ambientes I e II apresentaram menor número de indivíduos em relação ao ambiente III. Porém houve maior quantidade de indivíduos na classe 1 e menor na classe 2. Nota-se que a presença do incêndio reduziu o número de indivíduos, mas favoreceu aumento do número de indivíduos menores. Mesmo não tendo uma diferenciação na distribuição hipsométrica natural (distribuição normal), observa-se que há maior equilíbrio entre as classes 1 e 2 , demonstrando o efeito do fogo na estrutura vertical da comunidade.

Os mesmos ambientes I e II diferem do III quanto ao número médio de perfillhos por indivíduos, sendo também diferentes entre si. Já em relação à área basal, que também é significativa, os ambientes I e II são menores que o III. Esse fato demonstra que a presença do fogo aumenta a quantidade de perfilhos por indivíduos, não sendo suficiente para a área basal ser maior, já que a quantidade de indivíduos é menor. O efeito do fogo em relação aos perfilhos foi também identificado por Xavier et al. (2011).

\section{CONCLUSÃO}

O incêndio modificou a quantidade de indivíduos, além de aumentar significativamente o número de perfilhos por indivíduo, como consequência da morte da parte aérea deles, e de afetar a estrutura vertical da vegetação e a quantidade da área basal e, consequentemente, da biomassa aérea da comunidade. Porém, não foram identificadas alterações na estrutura horizontal e na composição florística entre os ambientes. Concluiu-se também que a rebrota foi a principal estratégia utilizada pelas espécies nos locais com ocorrência de incêndio

\section{STATUS DA SUBMISSÃO}

Recebido: 24 ago., 2014

Aceito: 15 maio, 2016 
AUTOR(ES) PARA CORRESPONDÊNCIA

\section{Milton Serpa de Meira Junior}

Departamento de Engenharia Florestal, Universidade de Brasília - UnB, Campus Darcy Ribeiro, Asa Norte, CP 04357, CEP 70910-900, Brasília, DF, Brasil

e-mail: milton.serpa@gmail.com

\section{REFERENNCIAS}

APG III. An update of the Angiosperm Phylogeny Group classification for the orders and families of flowering plants. Botanical Journal of the Linnean Society 2009; 161: 105-121.

Ávila GC, Souza DE. Incêndios Florestais no Parque Estadual do Biribiri e entorno imediato, entre 2007 e 2011 e suas relações com a presença humana. In: Congresso Brasileiro de Unidades de Conservação; 2012, Natal. Paraná: Fundação Grupo Boticário; 2012. p. 21.

Brower JE, Zar JH. Field and laboratory methods for general ecology. Dubuque: Win C. Brown Publishers; 1984. 226 p.

Cochrane M, Schulze MD. Fire as a recurrent event in tropical forests of the eastern Amazon: effects on forest structure, biomass, and species composition. Biotropica 1999; 31(1): 2-16.

Felfili JM, Rezende RP. Conceitos e métodos em fitossociologia. Brasília: Universidade Federal de Brasília; 2003. 68 p. Comunicações-técnicas Florestais.

Felfili JM, Silva-Júnior MC, Filgueiras TS, Nogueira PE. Comparison of cerrado (sensu stricto) vegetation in central Brazil. Ciencia e Cultura 1998; 50: 237-243.

Ferreira EB, Cavalcanti PP, Nogueira DA. ExpDes: experimental designs package. R package version (1.1.2). 2013.

Finol UH. Nuevos parametros a considerarse em el analisis estrutural de las selvas virgenes tripicales. Revista Florestal Venezuelana 1971; 14(21): 29-42.

Hoffmann WA. Post-burn reproduction of woody plants in a Neotropical savanna: the relative importance of sexual and vegetative reproduction. Journal of Applied Ecology 1998; 35(3): 422-433. http://dx.doi.org/10.1046/j.13652664.1998.00321.x.

Kauffman JB. Survival by sprouting following fire in tropical forest of the Eastern Amazon. Biotropica 1991; 23(3): 219-224. http://dx.doi.org/10.2307/2388198.

Klink CA, Solbrig OT. Efeito do fogo na biodiversidade de plantas do Cerrado. In: Sarmiento G, Cabido M, editores. Biodiversidad y funcionamento de pastizales y sabanas en América Latina. Mérida: Cyted y Cielat; 1996. p. 231-244.

Leitão Filho HF. A flora arbórea dos cerrados do Estado de São Paulo. Hoehnea 1992; 19: 151-163.
Lorenzi H. Árvores brasileiras: manual de identificação e cultivo de plantas arbóreas nativas do Brasil. Nova Odessa: Plantarium; 1992. 352 p.

Martins SV, Ribeiro GA, Silva-Junior WM, Nappo ME. Regeneração pós-fogo em um fragmento de floresta estacional semidecidual no município de Viçosa, MG. Ciência Florestal 2002; 12(1): 11-19.

Mendonça RC, Felfili JM, Walter BMT, Silva-Júnior MC, Rezende AV, Filgueiras TS et al. Flora vascular do cerrado. In: Sano SM, Almeida SP, editores. Cerrado: ambiente e flora. Brasília: Embrapa/CPAC; 1998. p. 289-556.

Miranda IS, Absy ML, Rebêlo GH. Community struture of Woody plants of Roraima Savannahs, Brazil. Plant Ecology 2002; 164(1): 109-123. http://dx.doi. org/10.1023/A:1021298328048.

Mueller-Dombois D, Ellenberg H. Aims and methods of vegetation ecology. New York: John Wiley \& Sons; $1974.547 \mathrm{p}$.

Nascimento MT. Rapid resprouting following fire in juveniles of Metrodorea pubescens (Rutaceae) in a Mesophytic forest in Central Brazil. Ciencia e Cultura 1996; 48: 182-183.

Neves SC, Abreu PAA, Fraga LMS. Fisiografia. In: Silva AC, Pedreira LCVSF, Abreu PAA, editores. Serra do Espinhaço Meridional: paisagens e ambientes. Belo Horizonte: $\mathrm{O}$ Lutador; 2005. p. 47-58.

Pereira IM, Gonzaga APD, Machado ELM, Oliveira MLR, Marques IC. Estrutura da vegetação colonizadora em ambiente degradado por extração de cascalho em Diamantina, MG. Pesquisa Florestal Brasileira 2015; 35(82): 77-88. http://dx.doi.org/10.4336/2015.pfb.35.82.769.

R Development Core Team. $R$ : a language and environment for statistical computing. Vienna: R Foundation for Statistical Computing; 2015 [citado em 2015 fev 21]. Disponível em: http://www.R-project.org/.

Ratter JA, Bridgewater S, Atkinson R, Ribeiro JF. Analysis of the floristic composition of the Brazilian cerrado vegetation II: comparison of the woody vegetation of 98 areas. Edinburgh Journal of Botany 1996; 53(02): 153-180. http://dx.doi.org/10.1017/S0960428600002821.

Ribeiro JF, Walter BMT. Fitofisionomias do cerrado. In: Sano S, Almeida S, editores. Cerrado: ambiente e flora. Planaltina: Embrapa-CPAC; 2008. p. 89-166.

Santiago J, Silva-Júnior MC, Lima L. Fitossociologia da vegetação arbórea na mata de galeria do Pitoco (IBGEDF), seis anos após fogo acidental. Scientia Forestalis 2005; 67: 64-77.

Santos MFV, Gutiérrez E, Vallejo R, Meunier IJ, Cillero D. Diversidade da vegetação pós-incêndio em terraços abandonados e ladeiras não cultivadas em Valença, Espanha. Revista Árvore 2003; 27(3): 399-405. http:// dx.doi.org/10.1590/S0100-67622003000300018. 
Silva-Júnior MC. 100 árvores do cerrado: guia de campo. Brasília: Rede de Sementes do Cerrado; 2005. 278 p.

Souza VC, Lorenzi H. Botânica sistemática: guia ilustrado para identificação das famílias de Angiospermas da flora brasileira, baseado em APG II. Nova Odessa: Plantarum; $2005.640 \mathrm{p}$.

Tannus JLS, Assis MA. A Composição de espécies vasculares de campo sujo e campo úmido em área de cerrado, Itirapina - SP, Brasil. Revista Brasileira de Botanica 2004; 27(3): 489-509. http://dx.doi.org/10.1590/S010084042004000300009 .
Xavier KRF, Andrade LA, Coelho MSE, Assis FNM, Fabricante JR. Impactos do fogo sobre o componente arbustivo-arbóreo de um remanescente de Floresta Ombrófila Aberta, Areia, Estado da Paraíba. Acta Scientiarum Biological Sciences 2009; 31(4): 407-413. http://dx.doi. org/10.4025/actascibiolsci.v31i4.4522.

Xavier KRF, Andrade LA, Fabricante JR, Coelho MSE, Assis FNM. Impactos pós-fogo na regeneração natural em um fragmento de floresta ombrófila aberta no município de Areia, Paraíba, Brasil. Revista Brasileira de Biociências 2011; 9(3): 257-264. 